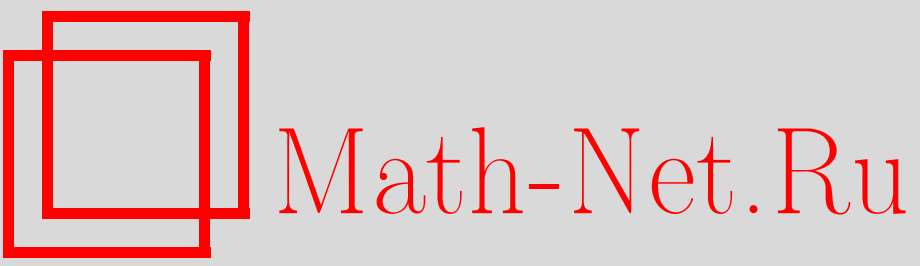

Б. С. Нахапетян, Сильная выпуклость давления для спиновых решетчатых систем, УМН, 1997, том 52, выпуск 2, 101-108

DOI: https://doi.org/10.4213/rm820

Использование Общероссийского математического портала Math-Net.Ru подразумевает, что вы прочитали и согласны с пользовательским соглашением

http://www . mathnet.ru/rus/agreement

Параметры загрузки:

IP : 54.162 .85 .209

26 апреля 2023 г., 11:03:46

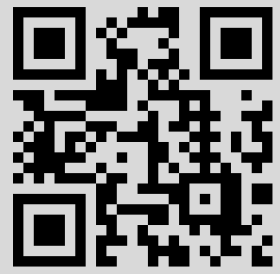




\title{
СИЛЬНАЯ ВЫПУКЛОСТЬ ДАВЛЕНИЯ ДЛЯ СПИНОВЫХ РЕШЕТЧАТЫХ СИСТЕМ
}

\author{
Б. С. НАХАПЕТян
}

\section{СОДЕРЖАНИЕ}

1. Введение . . . . . . . . . . . 101

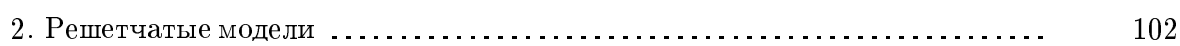

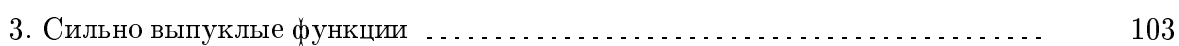

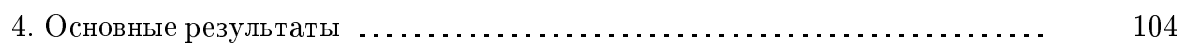

5. Оценки разностей гиббсовских плотностей с различными граничными усло-

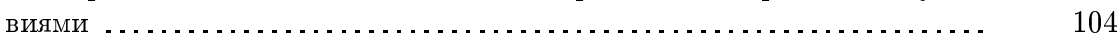

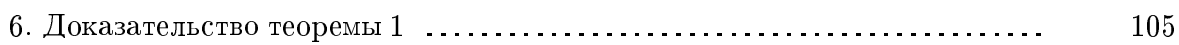

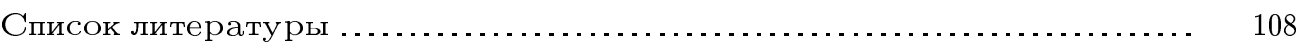

1. Введение. Полезные сведения об обшей структуре фазовых диаграмм статфизических моделей можно получить, изучая свойства давления как функции на соответствующем банаховом пространстве потенциалов. Свойство вьпуклости (а также строгой выпуклости) давления хорошо известно (см., например, [1]). Менее известно свойство сильной выпуклости давления, впервые установленное Р. Л. Добрушиным для непрерьвных систем с парным финитным потенциалом (см. [2]). Заметим, что из сильной выпуклости вытекает, в частности, отделимость от нуля второй производной давления (в случае ее сушествования) вдоль любого направления, задаваемого невырожденным потенциалом. В [2] Р. Л. Добрушиным был предложен новый подход к анализу свойств вьпуклости давления, основанный на одном вероятностном неравенстве, часто используемом в математической статистике. Указанный подход Р. Л. Добрушина был развит в работе [3], где для решетчатых систем с конечным пространством спинов было доказано свойство сильной вьпуклости давления и разъяснена связь этого свойства с проблемой убьвания коррелящий соответствующего гиббсовского случайного поля.

В настоящей заметке мы обобщаем результаты работы [3], показьвая, что свойство сильной выпуклости давления имеет место для решетчатых систем с обшим взаимодействием и стандартным пространством спинов с конечной мерой. То же свойство верно для вакуумных систем при менее ограничительных условиях на потенциал. 
2. Решетчатые модели. Пусть $\mathbb{Z}^{\nu}$ - целочисленная решетка, $X$ - стандартное пространство с конечной мерой $\mu, 0<\mu(X)<\infty$, и $\Phi$ - потенциал,

$$
\Phi: \bigcup_{J \in W} X^{J} \rightarrow \mathbb{R}^{1}
$$

где

$$
W=\left\{J \subset \mathbb{Z}^{\nu}:|J|<\infty\right\}, \quad X^{J}=\left\{x_{t}, t \in J\right\}, \quad x_{t} \in X, \quad t \in J, \quad J \in W .
$$

В дальнейшем будем рассматривать только транслящионно инвариантнье потенциалы. Положим

$$
\begin{gathered}
\|\Phi\|_{\gamma}=\sum_{J: O \in J \in W}|J|^{\gamma} \sup _{x \in X^{J}}|\Phi(x)|<\infty, \quad \gamma \in \mathbb{R}^{1}, \\
\mathscr{B}_{\gamma}=\left\{\Phi:\|\Phi\|_{\gamma}<\infty\right\} .
\end{gathered}
$$

Для любых $x \in X^{I}, y \in X^{V}$ через $x \vee y$ обозначим соответствующий элемент в $X^{I \cup Y}$. Введем гиббсовскую плотность в конечном сосуде $\Lambda$ и с граничными условиями $\bar{x} \in X^{\mathbb{Z}^{\nu} \backslash \Lambda}$ :

$$
\begin{gathered}
q_{\Lambda}^{\bar{x}, \Phi}(x)=\left(Z_{\Lambda}^{\Phi}(\bar{x})\right)^{-1} \exp \left\{-U_{\Lambda}^{\bar{x}, \Phi}(x)\right\}, \quad x \in X^{\Lambda}, \\
Z_{\Lambda}^{\Phi}(\bar{x})=\int_{X^{\Lambda}} \exp \left\{-U_{\Lambda}^{\bar{x}, \Phi}(x)\right\} \mu^{\Lambda}(d x),
\end{gathered}
$$

где

$$
U_{\Lambda}^{\bar{x}, \Phi}(x)=\sum_{\varnothing \neq J \subset \Lambda} \sum_{\widetilde{J} \subset \mathbb{Z}^{\nu} \backslash \Lambda} \Phi\left(x_{J} \vee \bar{x}_{\widetilde{J}}\right)
$$

есть потенциальная энергия на $X^{\Lambda}$ с граничными условиями $\bar{x} \in X^{\mathbb{Z}^{\nu} \backslash \Lambda}, x_{J}$ и $\bar{x}_{\widetilde{J}}-$ сужения конфигураций $x$ и $\bar{x}$ на множества $J$ и $\widetilde{J}$, соответственно.

Расссмотрим функцию

$$
\mathbf{P}_{\Lambda}^{\bar{x}}(\Phi)=|\Lambda|^{-1} \log Z_{\Lambda}^{\Phi}(\bar{x}), \quad \Phi \in \mathscr{B}_{1}, \quad \bar{x} \in X^{\mathbb{Z}^{\nu} \backslash \Lambda}
$$

Стандартными методами показьвается (см. [1], [4]), что для любой последовательности $\Lambda_{n}, n=1,2, \ldots, \Lambda_{n} \in W, \bigcup_{n} \Lambda_{n}=\mathbb{Z}^{\nu}$, стремящейся к бесконечности по Ван-Хову, существует предел (давление)

$$
\lim _{n \rightarrow \infty} \mathbf{P}_{\Lambda_{n}}^{\bar{x}_{n}}(\Phi)=\mathbf{P}(\Phi), \quad \Phi \in \mathscr{B}_{1}
$$

не зависяший от выбора последовательности $\Lambda_{n}$ и граничных условий $\bar{x}_{n} \in X^{\mathbb{Z}^{\nu} \backslash \Lambda_{n}}$, $n=1,2, \ldots$. 
Потенциал $\Phi \in \mathscr{B}_{1}$ назовем вырожденньм, если сушествует функция $F_{\{0\}}^{\bar{x}}$, $\bar{x} \in X^{\mathbb{Z}^{\nu} \backslash\{0\}}$, такая, что почти всюду по мере $\mu$

$$
U_{\{0\}}^{\bar{x}, \Phi}(x)=F_{\{0\}}^{\bar{x}}, \quad x \in X .
$$

Из (1) легко вытекает существование для любого $\Lambda \in W$ функции $F_{\Lambda}^{\bar{x}}, \bar{x} \in X^{\mathbb{Z}^{\nu} \backslash \Lambda}$, такой, что почти всюду по мере $\mu^{\Lambda}$

$$
U_{\Lambda}^{\bar{x}, \Phi}(x)=F_{\Lambda}^{\bar{x}}, \quad x \in X^{\Lambda}
$$

Вырожденные потенщиалы характеризуются тем, что совокупности гиббсовских распределений для потенциалов $\Phi$ и $\Phi+\Phi^{G}$, где $\Phi^{G}$ - вырожденный потенциал, совпадают.

Важньм классом невырожденных потенциалов является класс так назьваемых вакуумных потенциалов $\mathscr{B}_{0}^{\theta}$. Потенциал $\Phi \in \mathscr{B}_{0}$ назовем вакуумнылм, если в $X$ сушествует элемент $\theta$ ("вакуум") такой, что $\mu(\theta)=1$ и $\Phi(x)=0$ на каждой конфигурации $x \in X^{\Lambda}, \Lambda \in W$, содержаший вакуум, т.е. найдется $t \in \Lambda$ такое, что $x_{t}=\theta$.

3. Сильно выпуклые функции. Пусть $E$ - некоторое банахово пространство, $S \subseteq E$ - произвольное множество и $K \subseteq E$ - некоторьй конус. Функцию $h(x), x \in E$, назовем выпуклой на множестве $S$ по направлениям, принадлежашим конусу $K$, если при всех $x, x+l \in S$ таких, что $l \in K$, и при всех $\lambda \in[0,1]$

$$
\Delta_{x, l}^{\lambda} h \equiv \lambda h(x+l)+(1-\lambda) h(x)-h(x+\lambda l) \geqslant 0
$$

и строго вьлпуклой на $S$ по направлениям из конуса $K$, если в $(2)$ имеет место строгое неравенство.

Обычное определение выпуклости (строгой выпуклости) функции на некотором выпуклом множестве М эквивалентно следующему: функция $h$ выпукла (строго вьпукла) на множестве $M$ по направлениям, принадлежашим конусу $E$ (см. также [5]).

Назовем функцию $h(x)$ сильно выпуклой на множестве $S$ по направлениям из конуса $K$, если для каждого $r \in \mathbb{R}_{+}^{1}$ сушествует константа $C_{r}>0$ такая, что при всех $x, x+l \in S$, где $l \in K,\|l\| \leqslant r$, и при всех $\lambda \in[0,1]$

$$
\Delta_{x, l}^{\lambda} h \geqslant \lambda(1-\lambda) C_{r}\|l\|^{2}
$$

Если функция $h(x+\lambda l), x \in S, l \in K, x+\lambda l \in S, \lambda \in[\alpha, \beta] \subset[0,1],\|l\|<r$, дважды дифференщируема по $\lambda$, то из сильной вьпуклости $h$ следует, что

$$
\frac{\partial^{2} h(x+\lambda l)}{\partial \lambda^{2}} \geqslant C_{r}\|l\|^{2}, \quad \lambda \in[\alpha, \beta]
$$




\section{4. Основные результаты.}

Tеорема 1. Пусть $S \subset \mathscr{B}_{1}$ - компактное множество и $K \subseteq \mathscr{B}_{1}$ - некоторый конус. Если множество $K \cap E^{(1)}$, где $E^{(1)}$ - единичная сфера в $\mathscr{B}_{1}$, компактно и не содержит вырожденных потенциалов, то давление $\mathbf{P}(\Phi)$ сильно выпукло на $S$ по направлениям из конуса $K$.

Поскольку $\mathbf{P}\left(\Phi_{0}+\lambda \Phi\right)$ линейна при вырожденном потенциале, мы видим, что условие о том, чтобы $K \cap E^{(1)}$ не содержало вырожденных потенциалов, существенно.

Следуюшая теорема, касаюшаяся вакуумных потенщиалов, показывает справедливость гипотезы высказанной в [3].

Tеорема 2. Пусть $S \subset \mathscr{B}_{0}^{\theta}-$ компактное мноэсество и $K \subseteq \mathscr{B}_{0}^{\theta}-$ некоторый конус. Если множество $K \cap E^{(1)}$, где $E^{(1)}$ - единичная сфера в $\mathscr{B}_{0}^{\theta}$, компактно, то давление $\mathbf{P}(\Phi)$ сильно выпукло на $S$ по направлениям из конуса $K$.

5. Оценки разностей гиббсовских плотностей с различными граничными условиями. Для любого $\Lambda \in W$ положим

$$
\rho_{\Lambda}(t, s)=\frac{1}{2} \sup _{\bar{x}, \widetilde{x}} \int_{X}\left|q_{\{t\}}^{\bar{x}}(x)-q_{\{t\}}^{\widetilde{x}}(x)\right| \mu(d x),
$$

где $t, s \in \Lambda$, а верхная грань берется по всем конфигурациям $\bar{x}, \widetilde{x} \in X^{\Lambda \backslash\{t\}}$, отличающимся лишь в точке $s$. Далее, для любого $\Lambda \in W$ и $I \subset \Lambda$ будем обозначать

$$
\left(q_{\Lambda}^{\bar{x}}\right)_{I}(x)=\int_{X^{\Lambda \backslash I}} q_{\Lambda}^{\bar{x}}(x \vee y) \mu^{\Lambda \backslash I}(d y) .
$$

Лемма 1 (Р. Л. Добрушин [6], [7]). Для любого потенииала $\Phi \in \mathscr{B}_{1}$ имеет место неравенство

$$
\left|\left(q_{\Lambda \backslash V}^{\bar{x} \vee y}\right)_{I}(x)-\left(q_{\Lambda}^{\bar{x}}\right)_{I}(x)\right| \leqslant \sum_{t_{1} \in I} \sum_{t_{2} \in \Lambda} \ldots \sum_{t_{k-1} \in \Lambda} \sum_{t_{k} \in V} \rho_{\Lambda}\left(t_{1}, t_{2}\right) \rho_{\Lambda}\left(t_{2}, t_{3}\right) \cdots \rho_{\Lambda}\left(t_{k-1}, t_{k}\right),
$$

əде $I, V \in \Lambda, \Lambda \in W, I \cap V=\varnothing, x \in X^{I}, y \in X^{V}, \bar{x} \in X^{\mathbb{Z}^{\nu} \backslash \Lambda}$.

Используя метод коррелящионных уравнений, в [8] получена следующая оценка.

Лемма 2 (Б. С. Нахапетян, С.К. Погосян, [8]). Пусть $\Phi \in \mathscr{B}_{0}^{\theta}$. Тогда

$$
\left|\left(q_{\Lambda \backslash V}^{\bar{x}, y}\right)_{I}(x)-\left(q_{\Lambda}^{\bar{x}}\right)_{I}(x)\right| \leqslant 2^{|I|} B_{\Phi} \alpha_{\Lambda}(\delta(I, V)),
$$

где $B_{\Phi}$ - некоторая константа, зависящая от потенциала $\Phi$,

$$
\begin{aligned}
\alpha_{\Lambda}(d) & =\sum_{n=0}^{|\Lambda|}\left(\gamma_{\Phi}(z)\left(1+C_{\Phi}+\widetilde{C}_{\Phi}(z)\right)\right)^{n} r_{\Lambda}\left(\frac{d}{n+1}\right), \\
r_{\Lambda}(d) & =\frac{1}{\|\Phi\|_{0}} \sum_{\substack{O \in J \subset \Lambda \\
J \not \subset S(O, d)}} \sup _{\substack{ \\
x \in X^{J}}}|\Phi(x)|,
\end{aligned}
$$


$S(O, d)=\left\{t \in \mathbb{Z}^{\nu}: \delta(O, t) \leqslant d\right\}, d \in \mathbb{R}^{1}, \delta(\cdot, \cdot)$ - некоторая метрика на $\mathbb{Z}^{\nu}$,

$$
\begin{gathered}
z=\sup _{x \in X} z(x), \quad z(x)=e^{-\Phi(x)}, \quad x \in X, \\
\gamma_{\Phi}(z)=\frac{z \exp \left(\|\Phi\|_{0}\right)}{1+z \exp \left(\|\Phi\|_{0}\right)}, \quad C_{\Phi}=2 \exp \left(e^{\|\Phi\|_{0}}-1\right)-2, \\
\widetilde{C}_{\Phi}(z)=\beta(X) z e^{2\|\Phi\|_{0}}\left(e^{2\|\Phi\|_{0}}-1\right)\left(C_{\Phi}+2\right), \\
\beta(X)= \begin{cases}0, & \text { если }|X|=1, \\
1 & \text { в противном случае. }\end{cases}
\end{gathered}
$$

Далее, пусть $\operatorname{Cov}_{\Lambda}^{\bar{x}, \Phi}, \mathrm{D}_{\Lambda}^{\bar{x}, \Phi}, \mathrm{E}_{\Lambda}^{\bar{x}, \Phi}$ есть символы ковариации, дисперсии и математического ожидания относительно гиббсовского распределения в конечном сосуде $\Lambda$, с граничными условиями $\bar{x} \in X^{\mathbb{Z}^{\nu} \backslash \Lambda}$ и потенциалом $\Phi$.

Лемма 3. Пусть $\Phi \in \mathscr{B}_{1}$, тогда для любых функций $f_{I}(x), x \in X^{I}$, u $g_{V}(x)$, $x \in X^{V}, I, V \subset \Lambda, I \cap V=\varnothing$, имеет место неравенство

$$
\begin{aligned}
\left|\operatorname{Cov}_{\Lambda}^{\bar{x}, \Phi}\left(f_{I}, g_{V}\right)\right| & \leqslant \sup _{x \in X^{I}}\left|f_{I}(x)\right| \sup _{x \in X^{V}}\left|g_{V}(x)\right| \\
& \times \sum_{t_{1} \in I} \sum_{t_{2} \in \Lambda} \cdots \sum_{t_{k-1} \in \Lambda} \sum_{t_{k} \in V} \rho_{\Lambda}\left(t_{1}, t_{2}\right) \rho_{\Lambda}\left(t_{2}, t_{3}\right) \cdots \rho_{\Lambda}\left(t_{k-1}, t_{k}\right) .
\end{aligned}
$$

Если же $\Phi \in \mathscr{B}_{0}^{\theta}$, mo

$$
\left|\operatorname{Cov}_{\Lambda}^{\bar{x}, \Phi}\left(f_{I}, g_{V}\right)\right| \leqslant \sup _{x \in X^{I}}\left|f_{I}(x)\right| \sup _{x \in X^{V}}\left|g_{V}(x)\right| B_{\Phi} 2^{|I|} \alpha_{\Lambda}(\delta(I, V)) .
$$

6. Доказательство теоремы 1. В данном пункте мы приведем основные этапы доказательства теоремы 1 . Теорема 2 доказьвается аналогично с использованием оценок разностей гиббсовских плотностей с различными граничньми условиями из леммы 2.

Для $J, V \in \mathbb{Z}^{\nu}, J \in W, J \cap W=\varnothing$, положим

$$
\Phi_{J}^{\bar{x}}(x)=\sum_{\widetilde{J} \subset V} \Phi\left(x \vee \bar{x}_{\widetilde{J}}\right), \quad x \in X^{J}, \quad \bar{x} \in X^{V} .
$$

Тогда ясно, что

$$
\begin{aligned}
& U_{J}^{\bar{x}, \Phi}(x)=\sum_{J \subset I} \Phi_{J}^{\bar{x}}\left(x_{J}\right), \quad x \in X^{I}, \quad \bar{x} \in X^{\mathbb{Z}^{\nu} \backslash I}, \\
& U_{\{s\}}^{\bar{x}, \Phi}(x)=\Phi_{\{s\}}^{\bar{x}}(x), \quad s \in \mathbb{Z}^{\nu}, \quad x \in X, \quad \bar{x} \in X^{\mathbb{Z}^{\nu} \backslash\{s\} .}
\end{aligned}
$$

Основньм техническим приемом при доказательстве теоремы 1 является следуюшее неравенство

$$
\mathrm{D}_{\Lambda}^{\bar{x}, \Phi} g_{\Lambda} \geqslant \mathrm{E}_{\Lambda \backslash I}^{\bar{x}, \Phi} \mathrm{D}_{I}^{\bar{x}, \Phi}\left(g_{\Lambda} / y\right), \quad y \in X^{I}, \quad I \in \Lambda,
$$


где $g_{\Lambda}(x), x \in X^{\Lambda},-$ некоторая случайная величина.

Далее заметим, что для доказательства теоремы 1 достаточно построить последовательность $\nu$-мерных кубов $I_{n}, n=1,2, \ldots, \bigcup_{n} I_{n}=\mathbb{Z}^{\nu}$, такую, что

$$
\mathrm{D}_{I_{n}}^{\bar{x}_{n}, \Phi_{\lambda}} U_{I_{n}}^{\bar{x}_{n}, \Phi} \geqslant C\left|I_{n}\right|\|\Phi\|^{2}, \quad C>0,
$$

где $\Phi_{\lambda}=\Phi_{0}+\lambda \Phi, \Phi \in S \cap E^{(1)}, \lambda \in[0,1]$, а константа $C$ не зависит от граничных условий $\bar{x}_{n}, n=1,2, \ldots$, и от выбора потенциала из множества $S \cap E^{(1)}$.

Действительно, из (4) следует

$$
\frac{\partial^{2} \mathbf{P}_{I_{n}}^{\bar{x}_{n}}\left(\Phi_{\lambda}\right)}{\partial \lambda^{2}}=\frac{\mathrm{D}_{I_{n}}^{\bar{x}_{n}, \Phi_{\lambda}}\left(U_{I_{n}}^{\bar{x}_{n}, \Phi_{\lambda}}\right)}{\left|I_{n}\right|} \geqslant C\|\Phi\|^{2} .
$$

Отсюда получаем

$$
\Delta_{\Phi_{0}, \Phi}^{\lambda} \mathbf{P}_{I_{n}}^{\bar{x}_{n}} \geqslant \lambda(1-\lambda) C\|\Phi\|^{2} .
$$

Для доказательства (4) применим (3). При всех $I \subset \Lambda \in W$ можем написать

$$
\begin{aligned}
\mathrm{D}_{\Lambda}^{\bar{x}, \Phi_{\lambda}}\left(U_{\Lambda}^{\bar{x}, \Phi_{\lambda}}\right) & \geqslant \int_{X^{\Lambda \backslash I}} \mathrm{D}_{\Lambda}^{\bar{x}, \Phi_{\lambda}}\left(U_{I}^{\bar{x}, \Phi_{\lambda}} / y\right)\left(q_{\Lambda}^{\bar{x}, \Phi_{\lambda}}\right)_{I}(y) \mu^{I}(d y) \\
& =\int_{X^{\Lambda \backslash I}} \mathrm{D}_{I}^{\bar{x} \vee y, \Phi_{\lambda}} U_{I}^{\bar{x} \vee y, \Phi_{\lambda}}\left(q_{\Lambda}^{\bar{x}, \Phi_{\lambda}}\right)_{I}(y) \mu^{I}(d y) .
\end{aligned}
$$

Далее, для упрощения выкладок положим

$$
\mathrm{D}_{I}^{\bar{x}, \Phi_{\lambda}}=\overline{\mathrm{D}}, \quad \operatorname{Cov}_{I}^{\bar{x}, \Phi_{\lambda}}=\overline{\operatorname{Cov}}, \quad \bar{x} \vee y=\widetilde{x} .
$$

Имеем:

$$
\begin{aligned}
& \overline{\mathrm{D}}\left(U_{I}^{\bar{x}, \Phi_{\Lambda}}\right)= \overline{\mathrm{D}}\left(\sum_{s \in I} \Phi_{\{s\}}^{\tilde{x}}+\sum_{J \subset I,|J| \geqslant 2} \Phi_{J}^{\widetilde{x}}\right) \\
&=\sum_{s \in I}\left(\overline{\mathrm{D}} \Phi_{\{s\}}^{\widetilde{x}}+\right. \overline{\operatorname{Cov}}\left(\Phi_{\{s\}}^{\widetilde{x}}, \sum_{J \subset I,|J| \geqslant 2} \Phi_{J}^{\widetilde{x}}\right) \\
&\left.+\overline{\operatorname{Cov}}\left(\Phi_{\{s\}}^{\widetilde{x}}, \sum_{J \subset I,|J| \neq\{s\}} \Phi_{J}^{\widetilde{x}}\right)\right)+\overline{\mathrm{D}}\left(\sum_{J \subset I} \Phi_{J}^{\widetilde{x}}\right) \\
& \geqslant \sum_{s \in I}\left(\overline{\mathrm{D}} \Phi_{\{s\}}^{\widetilde{x}}+\overline{\operatorname{Cov}}\left(\Phi_{\{s\}}^{\widetilde{x}}, \sum_{J \subset I,|J| \geqslant 2} \Phi_{J}^{\widetilde{x}}\right)\right. \\
& \\
&\left.+\overline{\operatorname{Cov}}\left(\Phi_{\{s\}}^{\widetilde{x}}, \sum_{J \subset I,|J| \neq\{s\}} \Phi_{J}^{\widetilde{x}}\right)\right) .
\end{aligned}
$$

Пусть $\mathbb{Z}_{d}^{\nu}=\left\{t d: t \in \mathbb{Z}^{\nu}\right\}, d \in \mathbb{Z}_{+}^{\nu}$, и сопоставим каждому $s \in \mathbb{Z}^{\nu} \nu$-мерный куб

$$
b_{\{s\}}^{d}=\left\{t \in \mathbb{Z}^{\nu}: s^{(i)}-\frac{d}{2}<t^{(i)} \leqslant s^{(i)}+\frac{d}{2}, i=1, \ldots, \nu\right\} .
$$


Далее, пусть $I_{1}-$ некоторьй куб из пространства $\mathbb{Z}_{d}^{\nu}$. Положим

$$
I=\bigcup_{s \in I_{1}} b_{\{s\}}^{d}, \quad R_{r}^{S}=\sup _{\Phi \in S}\left\{R_{r}^{\Phi}\right\}, \quad R_{r}^{\Phi}=\sum_{\substack{J: O \in J \in W \\ \text { Diam } J \geqslant r}}|J| \sup _{x \in X^{J}}|\Phi(x)| .
$$

Нетрудно проверить, что для любого компактного $S \subset \mathscr{B}$ и любой возрастающей последовательности подмножеств $\left\{I_{n}\right\}$

$$
\lim _{n \rightarrow \infty} R_{\operatorname{Diam}\left(I_{n}\right)}^{S}=0
$$

Заметим, что при достаточно большом $d$

$$
\begin{aligned}
\sum_{t \in I_{1} \backslash\{s\}} \rho_{\Lambda}(t, s) & \leqslant C_{1} \sum_{\substack{t \in I_{1} \backslash\{s\}\\
}} \sum_{J: t, s \in J \in I_{1}} \sup _{x \in X^{J}}\left|\Phi_{\lambda}(x)\right| \\
& \leqslant C_{2} \sum_{\substack{J: t, s \in J \in W \\
\delta(t, s)>d}}|J| \sup _{x \in X^{J}}\left|\Phi_{\lambda}(x)\right| \leqslant C_{3} R_{d}^{S} .
\end{aligned}
$$

Из этого соотношения и леммы 3 следуют оценки

$$
\begin{aligned}
& \left|\overline{\operatorname{Cov}}\left(\Phi_{\{s\}}^{\widetilde{x}}, \sum_{J \subset I,|J| \geqslant 2} \Phi_{J}^{\widetilde{x}}\right)\right| \leqslant C_{4} R_{d}^{S}, \\
& \left|\overline{\operatorname{Cov}}\left(\Phi_{\{s\}}^{\widetilde{x}}, \sum_{J \subset I,|J| \neq\{s\}} \Phi_{J}^{\widetilde{x}}\right)\right| \leqslant C_{5} R_{d}^{S} .
\end{aligned}
$$

Таким образом, при достаточно большом, но фиксированном $d$ последние выражения малы. С другой стороны, из условия невырожденности потенщиала $\Phi$ следует существование множества $A_{\Phi} \subset \mathscr{B}_{1}$ такого, что $\mu\left(A_{\Phi}\right)>0$ и

$$
\inf _{x, z \in \Lambda}\left|\Phi_{\{s\}}^{\bar{x}}(x)-\Phi_{\{s\}}^{\bar{x}}(z)\right| \geqslant C>0
$$

Из компактности множества $S$ следует, что константа в правой части последнего неравенства не зависит от выбора направления $\Phi \in S \cap E^{(1)}$. Следовательно, при достаточно большом $d$

$$
\overline{\mathrm{D}}\left(U_{I}^{\bar{x}, \Phi_{\Lambda}}\right) \geqslant C|I|
$$

Пусть теперь $\widetilde{I}_{n} \subset \mathbb{Z}_{d}^{\nu}$-некоторая последовательность возрастающих $\nu$-мерных кубов с достаточно большим $d>0$. Полагая

$$
I_{n}=\bigcup_{s \in \widetilde{I}_{n}} b_{\{s\}}^{d} \subset \mathbb{Z}^{\nu}
$$

получаем искомую последовательность, для которой вьполнено (4). Теорема 1 доказана. 


\section{СПИСОК ЛИТЕРАТУРЫ}

[1] Georgii H. Gibbs measures and phase transitions. Berlin: Walter de Gruyter, 1988.

[2] Добрушин Р. Л., Минлос Р. А. Существование и непрерьвность давления в классической статистической физике // Теория вероятн. и ее примен. 1967. Т. 12. №4. С. 595-618.

[3] Добрушин Р. Л., Нахапетян Б.С. Сильная выпуклость давления для решетчатых систем классической статистической физики // ТМФ. 1974. Т. 20. № 2. С. 223-234.

[4] Nahapetian B. S. Limit Theorems and Some Applications in Statistical Physics. Stuttgart: Teubner-Texte zur Mathematik, 1991.

[5] Поляк Б. Т. Теоремы существования и сходимость минимизирующих последовательностей для задач на экстремум при наличии ограничений // Докл. АН СССР. 1966. Т. 166. № 2 . C. $287-290$.

[6] Добрушин Р. Л. Описание случайного поля при помощи условных вероятностей и условия его регулярности // Теория вероятн. и ее примен. 1968. Т. 2. №2. С. 201-229.

[7] Добрушин Р. Л. Задание систем случайных величин при помощи условных распределений // Теория вероятн. и ее примен. 1970. Т. 15. №3. С. 469-497.

[8] Nahapetian B.S., Pogosian S. K. Decay of correlations in classical lattice spin systems with vacuum // Journ. Contemp. Math. Anal. 1995. V. 30. №6. P. 29-49.

Институт Математики

Поступила в редакцию

Национальной Академии Наук Армении

15.09.1996 